Research Article

\title{
Deep Learning-Based Wavelet Threshold Function Optimization on Noise Reduction in Ultrasound Images
}

\author{
Zhuxiang Shen (D), Wei Li $\mathbb{D}$, and Hui Han \\ Department of Equipment, Yancheng First People's Hospital, Yancheng 224000, Jiangsu, China \\ Correspondence should be addressed to Zhuxiang Shen; 180120304@mail.dhu.edu.cn
}

Received 16 June 2021; Revised 31 July 2021; Accepted 18 August 2021; Published 26 August 2021

Academic Editor: Enas Abdulhay

Copyright ( 92021 Zhuxiang Shen et al. This is an open access article distributed under the Creative Commons Attribution License, which permits unrestricted use, distribution, and reproduction in any medium, provided the original work is properly cited.

To explore the utilization of the convolutional neural network (CNN) and wavelet transform in ultrasonic image denoising and the influence of the optimized wavelet threshold function (WTF) algorithm on image denoising, in this exploration, first, the imaging principle of ultrasound images is studied. Due to the limitation of the principle of ultrasound imaging, the inherent speckle noise will seriously affect the quality of ultrasound images. The denoising principle of the WTF based on the wavelet transform is analyzed. Based on the traditional threshold function algorithm, the optimized WTF algorithm is proposed and applied to the simulation experiment of ultrasound images. By comparing quantitatively and qualitatively with the traditional threshold function algorithm, the advantages of the optimized WTF algorithm are analyzed. The results suggest that the image is denoised by the optimized WTF. The mean square error (MSE), peak signal-to-noise ratio (PSNR), and structural similarity index measurement (SSIM) of the images are $20.796 \mathrm{~dB}, 34.294 \mathrm{~dB}$, and $0.672 \mathrm{~dB}$, respectively. The denoising effect is better than the traditional threshold function. It can denoise the image to the maximum extent without losing the image information. In addition, in this exploration, the optimized function is applied to the actual medical image processing, and the ultrasound images of arteries and kidneys are denoised separately. It is found that the quality of the denoised image is better than that of the original image, and the extraction of effective information is more accurate. In summary, the optimized WTF algorithm can not only remove a lot of noise but also obtain better visual effect. It has important value in assisting doctors in disease diagnosis, so it can be widely applied in clinics.

\section{Introduction}

The continuous development of medical imaging technology makes the medical imaging become an essential part of clinical diagnosis and efficacy evaluation. Doctors can intuitively acquire the feature information of lesions through images, then evaluate the disease more accurately to achieve early detection and diagnosis, and strive for the best therapeutic effect for patients [1]. Medical images can be divided into different imaging techniques according to different imaging modes. The recognized representative imaging techniques include magnetic resonance imaging (MRI), $\mathrm{X}$-ray, ultrasound, and nuclear medicine imaging [2]. Ultrasound imaging, as a widely used examination method in clinics, has the advantages of safety, noninvasiveness, convenience, and low cost. Especially in the observation of fetal growth and development in pregnant women and diagnosis of abdominal organ lesions, it has high clinical practical value as well as great development prospects [3].

Ultrasound imaging process is as follows. First, the ultrasound is transmitted through the device and reflected back to the receiving end of the device to complete a complete ultrasound imaging process through the interaction of tissues and organs [4]. At present, in the process of the clinical application of ultrasound technology, it is found that the intrinsic speckle noise will appear in the ultrasound image due to the interference characteristics of the ultrasound pulse, resulting in damage to the details of the medical ultrasound image, blurring of edge information, and seriously affecting the quality of the ultrasound image [5]. The interference of ultrasound imaging has a great impact on the diagnosis and follow-up processing of doctors. Therefore, 
from the clinical point of view, it is necessary to further explore the algorithm of removing speckle noise, so as to provide a theoretical basis for the accurate diagnosis of diseases by clinicians. Convolutional neural network (CNN) is an essential part in deep learning which is one of the earliest networks that uses the backpropagation algorithm to train effectively. CNN is a typical feedforward neural network and has a good performance in large-scale image processing, especially in the scene with similar information characteristics.

The precondition of image recognition is image denoising and image edge detection, which are also of great value to image postanalysis and processing. Through the image denoising process, the interference noise can be removed so that the image quality as well as the visual effect can be better [6]. In addition, image matching, segmentation, and edge detection, as well as other processing, are based on image denoising, so the quality of image denoising determines the follow-up processing of the whole image. Wavelet transform is a multiscale signal analysis method and widely used in many fields such as digital image processing and computer science. In the wavelet threshold denoising algorithm, the noise wavelet coefficients can be directly reduced. In denoising, the edge information of the image is effectively enhanced to achieve better enhancement effect [7]. In this exploration, on the basis of fully understanding the theoretical knowledge of the wavelet transform, the wavelet threshold denoising algorithm is optimized, and its application in ultrasound imaging technology (UIT) and its impact on imaging results are analyzed. It is not rare to apply the wavelet threshold algorithm to image processing, but the research of this algorithm is not deep in the field of medical imaging. In this exploration, from the perspective of image denoising, wavelet threshold denoising algorithm is applied in UIT, so as to obtain the key information of medical images and provide help for clinicians to read and follow up the diagnosis of diseases.

\section{Methods}

2.1. Principle of Medical Ultrasound Imaging. Medical ultrasound diagnosis technology originated in the 1940s or so. Ultrasound refers to sound waves with frequencies higher than $20 \mathrm{kHz}$, which have far exceeded the upper limit of human hearing. Ultrasound is widely used in the field of medical angiography because it is easy to carry media information and can be transmitted in any different media [8]. Moreover, it has good directivity and strong penetrating force. Medical ultrasound imaging technology (UIT) takes the ultrasonic signal as the carrier, takes the reflection principle of the ultrasonic signal as the theoretical basis, extracts the information from the reflected signal of the human body through processing, and finally completes the imaging processing. The probe transmits the ultrasonic signal to the human body, and then the signal propagates in the human tissue. Different tissues have different resistances to the ultrasonic signal. Ultrasound signals are reflected and scattered on the tissue surface, and then the echo signals are automatically received by the probe. After a series of signal enhancement processing, the final clearer and more detailed ultrasound images can be obtained. According to different characteristics of different tissues in vivo, combined with pathological knowledge and clinical experience, the spatial location, nature, and severity of the lesion can be accurately evaluated [9].

In the process of transmission, the sound wave will be attenuated due to sound absorption. The relationship between sound intensity and transmission distance can be expressed as

$$
I=I_{0} e^{(-\alpha z)}
$$

In (1), $I_{0}$ represents the original sound intensity of ultrasound, $\alpha$ is the attenuation coefficient of ultrasound, and $z$ is the propagation distance of ultrasound. Generally, the physical processes that cause acoustic attenuation include viscous loss, heat conduction loss, and various forms of molecular relaxation. Due to different physical processes of attenuation, the variation range of attenuation coefficient will be larger.

Ultrasound imaging is mainly accomplished by the reflection of ultrasound. Because the magnitude of the reflection coefficient and the intensity of the reflected sound wave are different, the contrast of the image is also produced. When ultrasound is injected vertically from one medium into another, the reflection coefficient can be expressed as

$$
R=\left(\frac{z_{1}-z_{2}}{z_{1}+z_{2}}\right) \text {. }
$$

In (2), $R$ is the attenuation coefficient of ultrasound, and $z_{1}$ and $z_{2}$ represent the acoustic resistance of two different media, respectively. The equation reveals that the greater the difference of acoustic resistance between the two media is, the more the reflectivity of the acoustic wave is. Therefore, for the complex imaging environment of human tissues and organs, the medium is inhomogeneous. The reflected sound wave is related to the difference of acoustic resistance between adjacent media and the shape of the target to be detected. The contact between different transmission media will form a reflection interface. According to the transmission characteristics of sound waves, different phenomena will occur when sound waves pass through different interfaces. For a small reflection interface whose reflection interface is less than the acoustic wavelength, sound waves will scatter and have anisotropy, and the energy of reflected sound waves will also be attenuated. For the large reflection interface whose reflection interface is larger than the wavelength of the acoustic wave, the reflection of the acoustic wave will occur, and the direction of reflection mainly depends on the incident direction.

Medical UIT can be divided into four types according to the principle of ultrasound imaging and different scanning methods [10]. The origin and application of A-mode ultrasonography in medical diagnosis are the earliest. However, due to the limitation of the imaging principle, the onedimensional waveform of A-mode ultrasound imaging is not intuitive enough. Therefore, after the appearance of B-mode ultrasound, A-mode ultrasound imaging is no longer used. 
B-mode UIT uses the ultrasound probe to transmit ultrasound to the human body, and then ultrasound signals reflected from human tissues are displayed by brightness modulation. In the two-dimensional grayscale image of B-mode ultrasound imaging, the brightness of the light spot reflects the strength of the echo signal. The brighter the light spot is, the stronger the echo signal is. Figure 1 shows the basic principle of the B-mode ultrasound imaging instrument. M-mode UIT uses slow scanning circuit technology to complete the diagnosis of the heart disease. Its main function is to measure the motor organs, so M-mode ultrasonography is used in the clinical diagnosis of the heart, fetal heart, and arteries and vessels. D-mode ultrasound diagnostic instrument is commonly referred to as Doppler ultrasound diagnostic instrument. Color Doppler imaging can monitor hemodynamic parameters in real time. According to the color coding, the direction, velocity, and nature of blood flow in the target position are judged [11].

2.2. Noise Models in Ultrasound Images. The noise in the ultrasound image mainly includes Gaussian noise caused by system instability, circuit noise in the imaging process, and speckle noise caused by the principle of ultrasound imaging itself. Spot noise can seriously affect the quality of the ultrasound image and interfere with artificial evaluation or machine recognition. It is now believed that the signals collected by UIT are divided into two parts: signals reflected by tissues in vivo with the interpretation value and noisy signals interfering with them. The noise signal includes additive noise and multiplicative noise. Multiplicative noise originates from the random scattering signal, which is related to the principle of ultrasonic imaging technology. Additive noise refers to the noise generated by the system. The initial signal obtained by the ultrasound imaging system is $f^{\text {pre }}$, and its general model can be expressed as follows:

$$
f^{\text {pre }}=g^{\text {pre }} n^{\text {pre }}+w^{\text {pre }} .
$$

In (3), pre represents the initial signal obtained by ultrasound imaging technology. The function $g^{\text {pre }}$ denotes the noise-free signal, $n^{\text {pre }}$ denotes the multiplicative noise, and $w^{\text {pre }}$ denotes the additive noise.

In the process of ultrasonic signal propagation, the phenomenon of random scattering occurs in a very small resolution, resulting in multiplicative noise $n^{\text {pre }}$. Multiplicative noise is the main part of the interference noise, while the effect of the additive noise can be neglected. Therefore, the model can be simplified as follows:

$$
f^{\mathrm{pre}}=g^{\mathrm{pre}} n^{\mathrm{pre}} .
$$

Multiplicative noise $n^{\text {pre }}$ is also called speckle noise because it can be visualized in the image by a speckle shape. The probability distribution of speckle noise varies with different ultrasound imaging systems because of different scattering in the unit resolution. In order to adapt to the grayscale display range of the screen of the ultrasound imaging system, the signals collected by the ultrasound imaging system are processed by logarithmic transformation. In this case, the

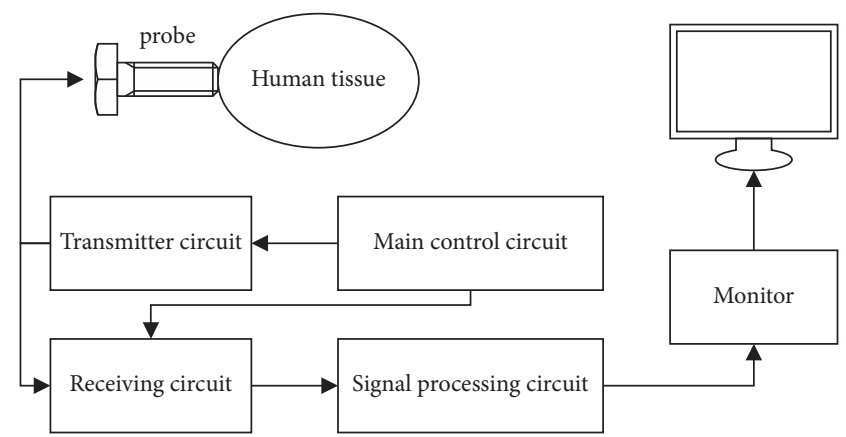

Figure 1: Basic principle diagram of the B-mode ultrasound imaging instrument.

multiplication equation (4) model will be changed into an additive model, which is expressed as

$$
\log \left(f^{\text {pre }}\right)=\log \left(g^{\text {pre }}\right)+\log \left(n^{\text {pre }}\right) .
$$

At this time, the signal $\log \left(f^{\text {pre }}\right)$ is the general medical ultrasound image. Log is equivalent to a compression function, which compresses the signals with a large range of changes to a smaller range. The noise model can not only be as close as possible to the actual distribution of the ultrasonic signal but also integrate with the corresponding denoising algorithm to make the denoising effect of the ultrasound image more satisfactory.

2.3. Image Denoising Based on the CNN. The network framework of low-illumination image processing based on the U-Net convolution network has three modules: preprocessing module, U-Net convolution network module, and superresolution reconstruction module. According to the RGB (red, green, and blue) color space model, the original raw image is input into four channels in the preprocessing module, and the dimension is reduced to $1 / 2$. Then, the black level is extracted, and the image is magnified at different ratios. The U-Net convolution network module realizes the image pixel semantic segmentation, and the output is 12 channels. There are 23 volume layers in the whole U-Net network. The left is the contraction part of capturing context information, and the right is the extension part of accurately locating segmentation results, called "contraction path" and "expansion path," respectively. The two paths form a shape that is similar to "U" shape symmetrically.

Due to the subpixel operation, when the image resolution is improved, it can be realized by recombining the pixels of the image. The subpixel convolution method of pixel recombining is used to improve the resolution without distortion. The process of subpixel convolution mainly includes recombining a single pixel on a multichannel feature map to a feature map. The subpixel convolution operation is carried out on the low-resolution image, and the final results are the high-resolution image.

2.4. Basic Principle of Wavelet Transform Denoising. The denoising of the image by wavelet transform means that, after decomposing the noise wavelet coefficients, the wavelet 
coefficients can be calculated which are infinitely close to the pure image wavelet coefficients. The wavelet coefficients are composed of pure wavelet coefficients $k_{i, j}$ and noise wavelet coefficients $p_{i, j}$. The sum of pure wavelet coefficients and noise wavelet coefficients is the wavelet coefficients $x_{i, j}$ of noise image decomposition. Through the threshold function, the noisy image is processed, and the wavelet coefficients $y_{i, j}$ after the threshold are obtained. If $y_{i, j}-k_{i, j} \approx 0$, the maximization of noise removal can be achieved, and a purer reconstructed image with wavelet coefficients can be obtained.

The principle of wavelet threshold denoising is to select the appropriate wavelet by using the change of wavelet. Through the multilevel decomposition of image noise, the corresponding high-frequency signal component and lowfrequency signal component are extracted from the decomposition wavelet coefficients. Combining with the corresponding threshold function, the high-frequency coefficients of the wavelet are thresholded. Effective lowfrequency signal coefficients of observable value are retained. The effective low-frequency signal and the threshold highfrequency signal wavelet coefficients are reconstructed to obtain the denoised image. Combining with the denoising evaluation index, the denoised image is quantitatively and qualitatively analyzed to evaluate the denoising effect and the quality of the denoised image. Because of the high correlation of the wavelet transform, the effective signal wavelet domain of the image can be concentrated in the wavelet coefficients with large coefficients, while the noise wavelet coefficients are randomly distributed in the whole domain. Through threshold calculation, satisfactory denoising effect can be achieved.

\subsection{Optimization of Wavelet Threshold Function (WTF)} Algorithms. In the wavelet transform denoising, the threshold function will directly affect the final denoising effect of the image. When the threshold value is small, the noise figure larger than the threshold value will be retained as an effective signal, which leads to little significance of denoising. The denoised image is still disturbed by many uncancelled noises. When the threshold value is too large, the effective information with small coefficients is treated as noise cancellation. Although the denoised image will be very smooth, more useful details will be lost in the denoising process.

The traditional WTF is a classical threshold function proposed by foreign researchers in 1994, also known as the unified threshold function [12]. From the theoretical point of view, it is proved that $M$ is used to represent the total number of wavelet coefficients in the corresponding wavelet domain, and $\sigma_{n}$ is the standard deviation of noise. The derived equation is expressed as

$$
T=\sigma_{n} \sqrt{2 \log M}
$$

Among the above threshold functions, threshold $T$ is greatly affected by the number of wavelet coefficients. When $M$ is larger, a larger threshold may remove some useful information with smaller coefficients [13]. Chang et al. proposed an optimal threshold selection method. This threshold function is derived from Bayesian maximum posteriori probability. $\sigma_{n}{ }^{2}$ is used to denote the variance of noise, and $\sigma_{g, j}$ is used to denote the standard deviation of the noise-free image $g$ in layer $j$ in the wavelet domain. $\alpha_{j}$ is an adjustable coefficient, that is, the adjustable coefficient in the $j$-layer of the wavelet domain, usually $\alpha_{j}=1$. Its equation is expressed as

$$
T=\alpha_{j}\left(\frac{\sigma_{n}^{2}}{\sigma_{g \cdot j}}\right) .
$$

In this exploration, if a new threshold function is proposed based on equation (6) to weaken the effect of the total number $M$ on the threshold, the effect of the wavelet function will be better. Therefore, based on the characteristics of the wavelet transform, the noise in the image is dense in the high-frequency region, so the threshold in the high-frequency region should be greater than that in the low-frequency region. In equation (7), on the basis of variance ratio $\sigma_{n}^{2} / \sigma_{g . j}$, the adjustable coefficient $\alpha_{j}$ should choose the appropriate value according to the wavelet coefficients of each layer; that is, the higher the number of layers is, the smaller the adjustable coefficient is. Therefore, in this exploration, a more refined WTF is proposed. $\sigma_{n}$ is used to represent the variance of noise, and $\sigma_{g, j}$ is used to represent the standard deviation of the noise-free image $g$ in layer $j$ in the wavelet domain. The adjustable parameters accord with $k_{1}+k_{2}=1$, and the equation is expressed as follows:

$$
T=\alpha_{j}\left(k_{1} \sigma_{n} \sqrt{2 \log M}+\frac{k_{2} \sigma_{n}^{2}}{\sigma_{g \cdot j}}\right) .
$$

In equation (8), the adjustment coefficient $\alpha_{j}$ of layer $j$ in the wavelet domain is $1 / 2^{j-1}, j=1,2, \ldots, J$. $J$ denotes the maximum level of wavelet decomposition. The WTF of equation (8) can be changed to

$$
T=\frac{1}{2^{j-1}}\left(k_{1} \sigma_{n} \sqrt{2 \log M}+\frac{k_{2} \sigma_{n}^{2}}{\sigma_{g \cdot j}}\right) .
$$

2.6. Evaluation Index and the Method of Denoising Effect. Qualitative evaluation: after image denoising, it is necessary to consider the effect and accuracy of the image. Since the effect of image processing affects the extraction and analysis of image information, it is particularly important to evaluate the effect of image denoising. First, the direct evaluation of the image is observed through naked eyes, and the image usually becomes blurred under the interference of noise. Therefore, the quality of image denoising can be roughly judged by human visual observation alone. However, subjective evaluation has some limitations. It can only directly reflect the clarity of the image and cannot extract and analyze the regional information of the image. According to the statistics of human visual sensory acuity, the criteria of subjective qualitative images are divided into five levels, and the specific indicators of each level are shown in Table 1. 
TABLE 1: Evaluation indexes of image denoising.

\begin{tabular}{lcc}
\hline Evaluation level & Relative scale & Decision scale \\
\hline 1 & The best image set is obtained & Very good \\
2 & Better than average of the image set & Preferable \\
3 & Average level of the image set & Common \\
4 & Lower than image set average & Poor \\
5 & The worst image concentration & Very bad \\
\hline
\end{tabular}

Quantitative evaluation: after preliminary qualitative evaluation, it is usually necessary to qualitatively evaluate the processed image to judge the quality of the denoising effect. First, the mean square error (MSE) of the image is the difference between the pure image and the processed image pixels. The magnitude of mean square deviation can reflect the degree of image distortion. It is considered that the larger the value of MSE is, the stronger the denoising power is. Then, peak signal-to-noise ratio (PSNR) is one of the methods widely used in image quality evaluation. The bigger the PSNR is, the better the image quality is. However, due to the limited sensitivity of personal visual effects, images with poor naked eye evaluation may appear. However, the relative coefficient of PSNR is relatively high. Structural similarity index measurement (SSIM) is an index to evaluate the image from the aspects of structure, brightness, and contrast $[14,15]$.

\section{Results}

3.1. Simulation Experiments and Results. To quantitatively evaluate the denoising effect of the algorithm proposed in this exploration, the evaluation experiment of the simulated image is carried out first. The original image selected in this exploration is shown in Figure 2(a). Hard threshold function denoising, soft threshold function denoising, compromise function denoising, and the optimized wavelet function threshold denoising are carried out, respectively, as shown in Figures 2(b)-2(e), respectively. The denoising effect of the hard threshold and soft threshold is different from that of the improved threshold function. In this paper, the optimized threshold expression is better than the traditional filter denoising for the noisy image, and the improved threshold denoising function is also better than other WTF algorithms under different noise variances.

In the simulation experiment, the basic threshold function and the optimized WTF are used to denoise the image. From the perspective of quantitative analysis through naked eye observation, the denoising effect of the hard threshold function and soft threshold function is slightly worse than that of the optimized WTF. However, further quantitative evaluation is needed to analyze the effect of denoising. In this exploration, the denoising effect of the threshold function is evaluated comprehensively by PSNR, MSE, and SSIM. Quantitative comparison of various threshold functions is shown in Table 2.

From the results of each index in the table, the noise removal situation and the denoising effect of various denoising functions can be analyzed. The denoising effect of the hard threshold function is better than that of the soft threshold function but lower than that of the compromise threshold function. The denoising effect of the optimized WTF in this exploration is better than that of the traditional threshold function.

3.2. Application Results of the CNN and WTF in Ultrasonic Medical Image Denoising. In order to fully analyze the denoising effect of the $\mathrm{CNN}$ and the optimized wavelet function algorithm, the ultrasound images of two different parts of arteries and kidneys are selected and analyzed. The contrast of artery tissue before and after denoising by the WTF is shown in Figures 3(a) and 3(b), and the effect of the kidney before and after denoising is shown in Figures 3(c) and $3(\mathrm{~d})$. Figure 3 suggests that compared with the original image, the medical ultrasound image processed by the wavelet threshold algorithm has significantly reduced the impact of noise around the target and has better imaging quality and clearer effect, whether it is the detailed imaging of the artery or the imaging of organs such as kidney tissue.

\section{Discussion}

The improvement of modern medical technology makes UIT play a vital role in clinical diagnosis and disease treatment. However, due to the limitation of the principle of ultrasound imaging, speckle noise is unavoidable in medical ultrasound images, which seriously affects the quality of ultrasound images and brings great interference to the diagnosis of doctors. Therefore, removing image noise as a key link in image processing can extract the original image and better analyze the image. The removal of speckle noise not only affects the result of image analysis and diagnosis but also is the basis of the development of the automatic diagnosis technology of the ultrasound imaging instrument in the later stage.

As a mathematical processing tool, wavelet transform can divide the data that need to be analyzed into different frequency components. With the development of the theory of wavelet transform, wavelet transform has become one of the commonly used tools in many scientific fields. The present principle of the wavelet transform has been widely used in the field of image denoising. The method of wavelet denoising is simple, and the quality of image processing is better. WTF algorithm is not only simple to calculate but also easy to complete, so it has been widely used in wavelet denoising. Aiming at the problem of medical ultrasound image noise removal, an optimized denoising algorithm is proposed in this exploration, i.e., the optimized WTF denoising algorithm. Three objective indexes, including MSE, PSNR, and SSIM, are selected to evaluate the denoising 


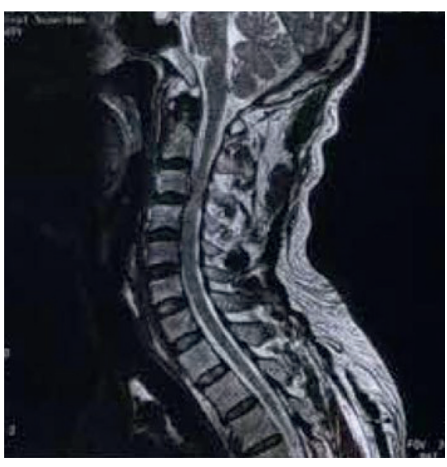

(a)

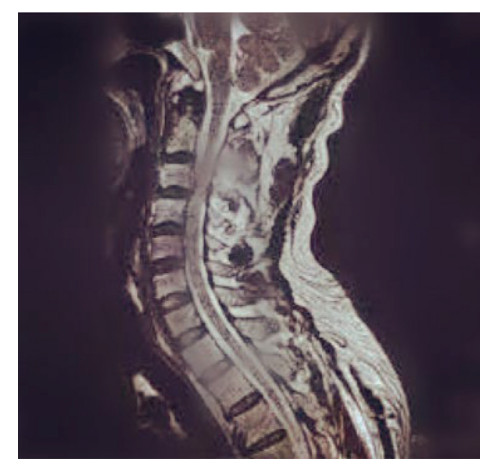

(b)

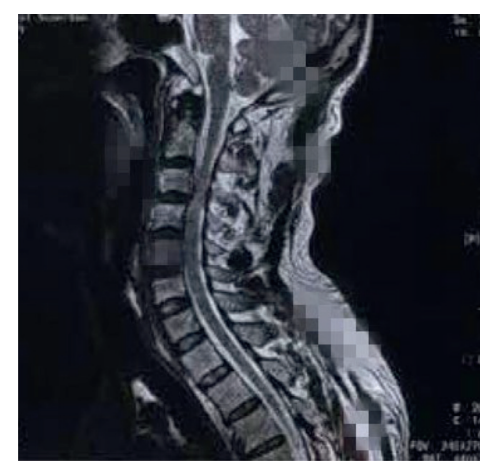

(c)

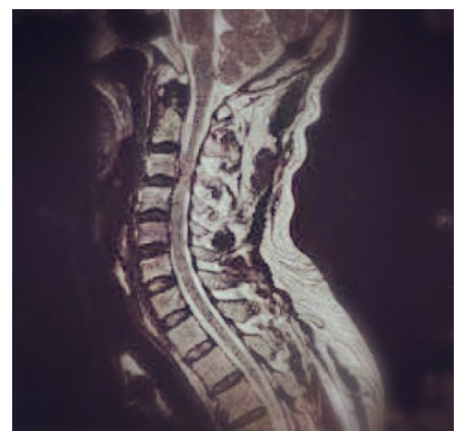

(d)

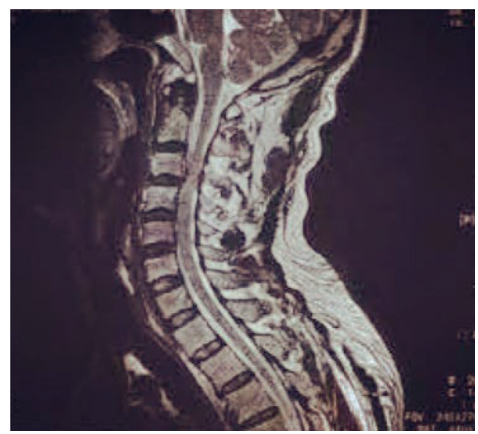

(e)

FIGURE 2: Denoising comparison of different thresholding functions of images: (a) original image; (b) hard threshold function denoising; (c) soft threshold function denoising; (d) compromise function denoising; (e) optimized wavelet function threshold denoising in this exploration.

TABLE 2: Quantitative comparison of various threshold functions for denoising (dB).

\begin{tabular}{lccc}
\hline Functional model & MSE & PSNR & SSIM \\
\hline Original image & 30.875 & 32.895 & 0.416 \\
Hard threshold function denoising & 22.439 & 34.608 & 0.636 \\
Soft threshold function denoising & 24.326 & 34.175 & 0.576 \\
Median function denoising & 22.359 & 34.640 & 0.636 \\
Optimized wavelet function threshold denoising & 20.796 & 34.294 & 0.672 \\
\hline
\end{tabular}

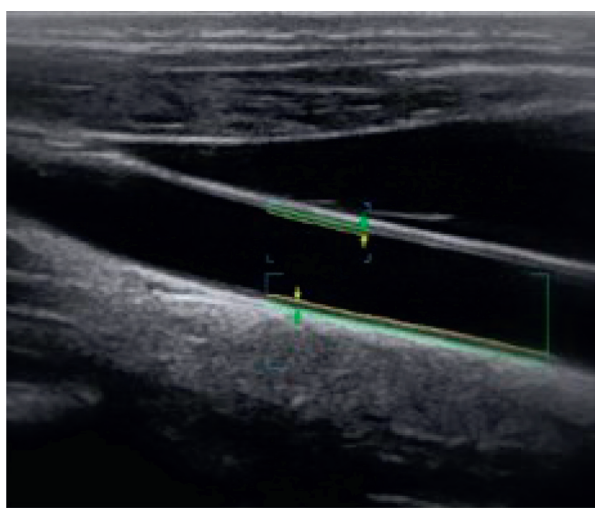

(a)

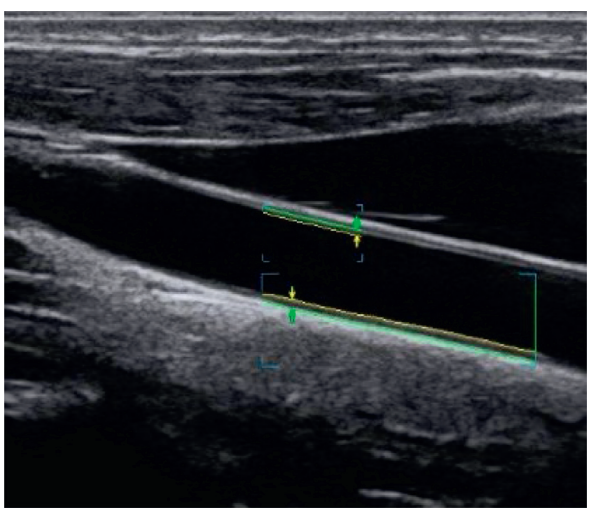

(b)

FIgUre 3: Continued. 


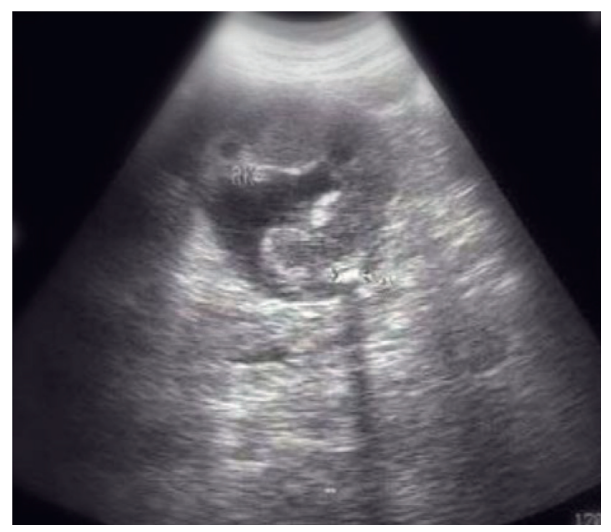

(c)

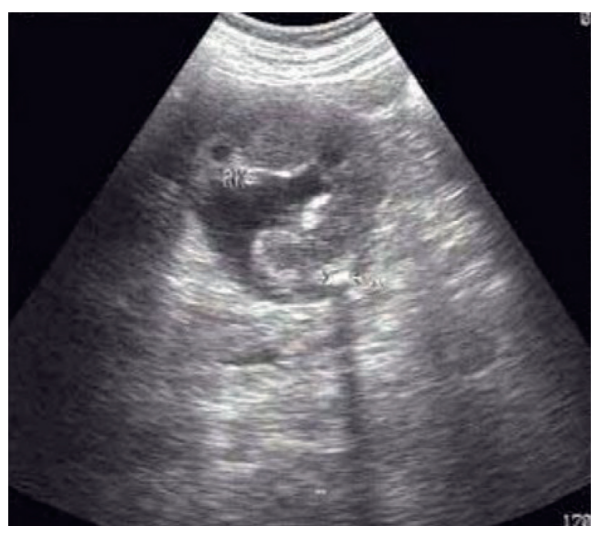

(d)

FIgURE 3: The contrast of the kidney before and after denoising: (a) artery tissue original image; (b) artery tissue denoised image; (c) kidney original image; (d) kidney denoised image.

effect of the image. In addition, the image denoising method based on deep learning is better than the traditional denoising method in the details and quality evaluation of image denoising. However, with the deepening of the network model and the improvement of the algorithm, there are some technical problems, such as gradient explosion, model optimization, and convergence. Based on deep learning, the problem of image denoising and enhancement is studied on the U-Net convolutional network.

Many scholars have confirmed that the wavelet threshold algorithm has a strong denoising effect in the remote sensing image, fuzzy video image processing, etc., which is consistent with the conclusion of this paper. The simulation results show that the optimized WTF is superior to other traditional thresholding functions in all aspects. It can denoise the image as much as possible without losing the image information. In addition, in this exploration, the optimized function is applied to the actual medical image processing, so as to denoise the arterial tissue and kidney, respectively. The results show that the quality of the denoised image is better than that of the original image, and the extraction of effective information is more accurate. In summary, the optimized WTF algorithm can obtain better visual effect while removing a lot of noise. It has important value in assisting doctors in disease diagnosis and can be widely used in clinics. In this exploration, the focus of the research is to remove the speckle noise in the image, focusing on the optimization of the WTF algorithm, while the optimization of the bilateral filtering algorithm is not deep enough, which needs to be further explored in the future research.

\section{Data Availability}

The data used to support the findings of this study are available from the corresponding author upon request.

\section{Conflicts of Interest}

The authors declare no conflicts of interest.

\section{References}

[1] W. Chen, X. Ding, J. Chen, and X. Liu, "The parameter selection method of wavelet threshold denoising in ultrasonic ranging signal process," Chinese Journal of Sensors and Actuators, vol. 30, no. 3, pp. 407-411, 2017.

[2] O. E. B'Charri, R. Latif, K. Elmansouri, A. Abenaou, and W. Jenkal, "ECG signal performance de-noising assessment based on threshold tuning of dual-tree wavelet transform," BioMedical Engineering Online, vol. 16, no. 1, 26 pages, 2017.

[3] F. Xie, C. Xiao, R. Liu, and L. Zhang, "Multi-threshold denoising of electrical imaging logging data based on the wavelet packet transform," Journal of Geophysics and Engineering, vol. 14, no. 4, pp. 900-908, 2017.

[4] Z. Lin, X. Zhou, Z. Wang, and C. Tan, “A nonmodel dual-tree wavelet thresholding for image denoising through noise variance optimization based on improved chaotic drosophila algorithm," International Journal of Pattern Recognition and Artificial Intelligence, vol. 31, no. 8, 25 pages, 2017.

[5] C. Shen, Y. Ding, J. Tang, X. Xu, and F. Guo, "An ameliorated prediction of drug-target interactions based on multi-scale discrete wavelet transform and network features," International Journal of Molecular Sciences, vol. 18, no. 8, 781 pages, 2017.

[6] J. Zhao, J.-S. Lee, H. Xu et al., "Scanner-dependent threshold estimation of wavelet denoising for small-animal PET," IEEE Transactions on Nuclear Science, vol. 64, no. 1, pp. 705-712, 2017.

[7] K. Hu, S. Liu, Y. Zhang et al., "Automatic segmentation of dermoscopy images using saliency combined with adaptive thresholding based on wavelet transform," Multimedia Tools and Applications, vol. 24, no. 6, pp. 1-18, 2019.

[8] H. Liu, W. Wang, C. Xiang, L. Han, and H. Nie, "A de-noising method using the improved wavelet threshold function based on noise variance estimation," Mechanical Systems and Signal Processing, vol. 99, no. 5, pp. 30-46, 2018.

[9] J. Shi, J. Liu, and L. Gongqi, "Research on wavelet threshold denoising method for power equipment's infrared image," Optical Technique, vol. 43, no. 2, pp. 162-165, 2017.

[10] K. Nagarathinam and R. S. Kathavarayan, "Moving shadow detection based on stationary wavelet transform and Zernike moments," IET Computer Vision, vol. 12, no. 6, pp. 787-795, 2018. 
[11] M. Risnandar and M. Aritsugi, "Deblocking artifact of satellite image based on adaptive soft-threshold anisotropic filter using wavelet," IEICE-Transactions on Info and Systems, vol. E101.D, no. 6, pp. 1605-1620, 2018.

[12] J.-j. Jiang, L.-r. Bu, X.-q. Wang et al., "Clicks classification of sperm whale and long-finned pilot whale based on continuous wavelet transform and artificial neural network," Applied Acoustics, vol. 141, pp. 26-34, 2018.

[13] R. N. Vargas, "Seismic trace noise reduction by wavelets and double threshold estimation," IET Signal Processing, vol. 11, no. 9, pp. 1069-1075, 2018.

[14] J. P. Marques, C. Lazaro, A. P. Morais, and G. Cardoso, "A reliable setting-free technique for power transformer protection based on wavelet transform," Electric Power Systems Research, vol. 162, no. 8, pp. 161-168, 2018.

[15] X. Wang and L. Xu, "Wavelet-based short-term forecasting with improved threshold recognition for urban expressway traffic conditions," IET Intelligent Transport Systems, vol. 12, no. 6 , pp. $463-473,2018$. 